\title{
Predictive Trajectory-Based Mobile Data Gathering Scheme for Wireless Sensor Networks
}

\author{
Fan Chao $\left(\mathbb{D},{ }^{1,2}\right.$ Zhiqin He $\mathbb{D},{ }^{1}$ Renkuan Feng $\mathbb{D},{ }^{1}$ Xiao Wang $\mathbb{D},{ }^{1}$ Xiangping Chen $\left(\mathbb{D},{ }^{1}\right.$ \\ Changqi Li $\mathbb{D})^{1}$ and Ying Yang $\mathbb{D}^{1}$ \\ ${ }^{1}$ School of Electrical Engineering Guizhou University, Guiyang 550025, China \\ ${ }^{2}$ School of Management Harbin Institute of Technology, Harbin 150000, China \\ Correspondence should be addressed to Zhiqin He; 641443416@qq.com
}

Received 30 March 2020; Revised 15 December 2020; Accepted 24 December 2020; Published 6 January 2021

Academic Editor: Fran ois P r s

Copyright (c) 2021 Fan Chao et al. This is an open access article distributed under the Creative Commons Attribution License, which permits unrestricted use, distribution, and reproduction in any medium, provided the original work is properly cited.

Tradition wireless sensor networks (WSNs) transmit data by single or multiple hops. However, some sensor nodes (SNs) close to a static base station forward data more frequently than others, which results in the problem of energy holes and makes networks fragile. One promising solution is to use a mobile node as a mobile sink (MS), which is especially useful in energy-constrained networks. In these applications, the tour planning of MS is a key to guarantee the network performance. In this paper, a novel strategy is proposed to reduce the latency of mobile data gathering in a WSN while the routing strategies and tour planning of MS are jointly optimized. First, the issue of network coverage is discussed before the appropriate number of clusters being calculated. A dynamic clustering scheme is then developed where a virtual cluster center is defined as the MS sojourn for data collection. Afterwards, a tour planning of MS based on prediction is proposed subject to minimizing the traveling distance to collect data. The proposed method is simulated in a MATLAB platform to show the overall performance of the developed system. Furthermore, the physical tests on a test rig are also carried out where a small WSN based on an unmanned aerial vehicle (UAV) is developed in our laboratory. The test results validate the feasibility and effectiveness of the method proposed.

\section{Introduction}

In recent years, the prosperous development in the Internet of Things has been validated where wireless sensor networks (WSNs) become ubiquitous. WSNs are widely used in the fields, such as intelligent transportation, agriculture, medical treatment, aerospace exploration, and other emerging applications, whereas they can contain a large number of static and mobile nodes in a self-organizing way [1-3]. Traditionally, a sink node in a WSN is fixed where the other sensor nodes (SNs) transmit data to the sink through either singlehop or multihop communication. Thus, the SNs close to the sink tend to carry more data transmission or forwarding tasks which leads to unbalanced energy consumption and results in poor data delivery in networks. The issues of energy holes and data collision may occur in such applications [4-7].

Mobile data gathering is regarded as a promising solution to tackle the problems aforementioned. In applications, mobile sinks (MSs) as data collectors gather sensing data in an efficient manner [8-15]. The issues associated with the tour planning of MS have been actively discussed in the existing literature that is crucially important to determine the performance of networks. The concern of energy consumption due to long-distance transmission is discussed in [16]. A maximum cache mechanism is proposed to enhance the transmission capability by adopting MS. In [17], an architecture of single-hop with single MS for mobile data 
gathering has been investigated. The proposed scheme has the benefit of energy-saving in line with the optimal tour trajectory of MS. In the study, a heuristic optimization algorithm is employed. A joint scheme for both charging and tour planning in a MS-based WSN is proposed in [18]. Energy consumption of each sensor is leveraged which in turn mitigates energy exhaustion. To strike the balance between energy consumption and latency of data gathering, an algorithm to find the optimal number and position of cluster head nodes for data collection is proposed in [19]. The simulation results verify the effectiveness of the scheme in favor of adopting the optimal path length of MS. In order to create a robust WSN, clustering techniques are also important for energy-saving and delay reduction where SNs are partitioned into subnetworks. Taking into account the kinematic constraints of mobile nodes of similar vehicles, in [20], a mobile data acquisition algorithm based on clustering Dubins smooth curve is proposed. Aiming at the problem of sensor node data aggregation and node energy imbalance, as well as the space problem being often ignored, a heuristic search algorithm (HLSA) is proposed in [21]. A dynamic clustering algorithm to divide the $\mathrm{SNs}$ into clusters is employed in [22]. By using the clustering mechanism, the energy efficiency and packet reception rate are improved. Our previous work has also shown the importance of sensor clustering in WSNs $[23,24]$. A WSN with respect to mobile data gathering where the work has a special focus on clustering mechanism before mobile data gathering is committed by the MS is developed in [23]. A data gathering scheme by using multiple mobile sink nodes is proposed in [24]. The average path length is dramatically shortened in this way, thereby reducing energy expenditure.

As an extension of the recent work, this study includes the main contributions as follows:

(1) We investigate the data gathering mechanism by considering energy constraints in a WSN system where the path length is minimized.

(2) The network coverage is discussed and formulated to an optimization problem. As a result, suitable node numbers are defined to strike the balance of the WSN scale and network coverage.

(3) A predicted trajectory is developed to assist the tour planning of a MS. In this manner, the overall data gathering is divided into several stages. This mechanism gives a chance to the MS to be recharged at the end of each stage to prevent energy exhaustion for a MS with insufficient power while heavy data load being required.

(4) Besides the extensive simulation on MATLAB, the trail tests are committed to verify the effectiveness of the proposed scheme where an unmanned aerial vehicle (UAV) serving as a MS is employed in a WSN.

The rest of this paper is organized as follows. Section 2 presents the systematic configuration with extensive discussion on energy constraints associated with MS traveling, activities, and the network architecture. Section 3 develops the optimization formulation for maximising network coverage while minimizing energy expenditure and path length for each cycle. Simulation setup and trail tests are both presented in Section 4 following the outcome demonstration and analysis corresponding to them. The key conclusions are drawn in Section 5.

\section{Problem Formulation}

The sensor network consists of a base station, a certain number of SNs, and a MS. After the sensors acquire data from the sensing field, they wait to be polled by the MS to deliver the information in each round. The WSN is shown in Figure 1.

It is assumed that there are $\mathrm{N}$ SNs in the network, $N=$ $\{1, \ldots \ldots, n\}, i \neq j, i, \quad j \in N ; V$ represents a virtual cluster, $V=$ $\{1, \ldots, v\}$; and trajectory between the virtual cluster head nodes is $M=\{V(i, j) i \neq j\}$. Let $d_{i j}$ represent the Euclidean distance between the two virtual cluster head and ley $x_{i j}^{m}$ indicate whether $V(i, j)$ is included in the tour of the MS. We have the following equation:

$$
x_{i j} \begin{cases}1, & \text { if data gathering tour contains } V(i, j), \\ 0, & \text { otherwise. }\end{cases}
$$

A MS gathers data periodically. The overall time in each cycle contains two segments: traveling and sojourn time [25]. Traveling time also includes two parts. One is in line with traversing time from one cluster to another. The second part is relevant to returning to the base station. Moreover, since the MS has the duty to poll the cluster head nodes, it stays on the cluster head nodes until completing data collection.

2.1. Traveling Time. In one cycle, the tour length can be calculated by

$$
D=\sum_{i=1}^{V} \sum_{j=1}^{V} x_{i j} d_{i j} .
$$

The total moving time is then calculated by

$$
t_{m}=\frac{D}{v}
$$

where $v$ is the moving speed.

2.2. Sojourn Time. In a cycle, the MS reaches sensor node $i$; the amount of data is $C_{i} ; \tau_{i}$ is staying time at each rendezvous point; $G$ represents the transmission rate. Therefore, there is a relationship as follows:

$$
\tau_{i}=\frac{C_{i}}{G} .
$$




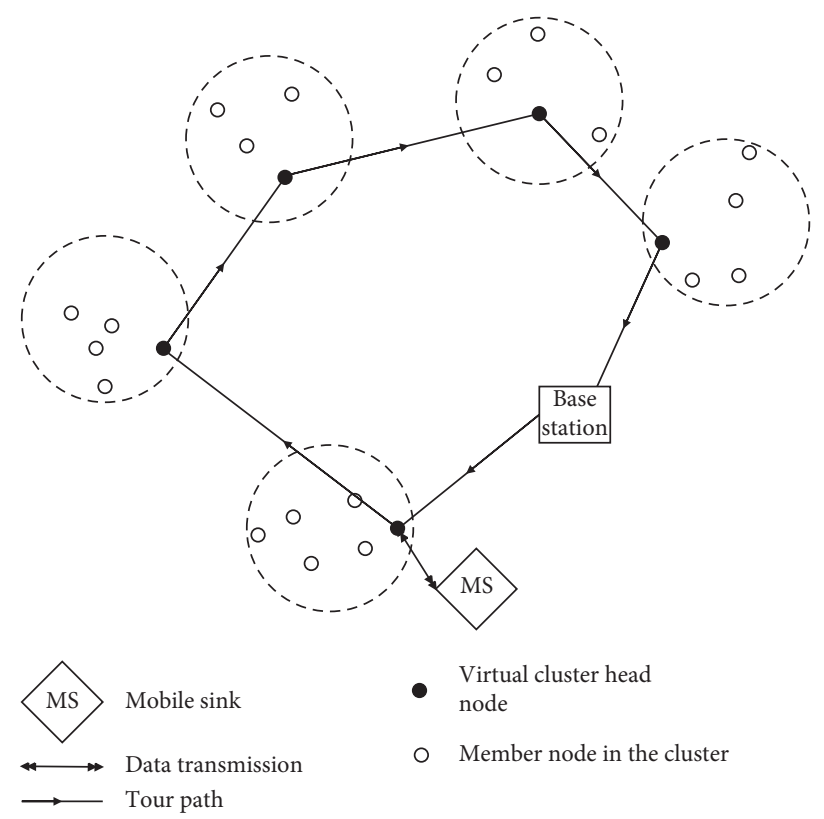

FIGURE 1: A wireless sensor network.

According to equations (3) and (4), the period of a cycle is obtained by

$$
T=t_{m}+\sum_{i=1}^{v} \tau_{i} .
$$

The tour planning is formulated to an optimization problem with the following equations:

$$
\begin{gathered}
\text { Minimize } t_{m}+\sum_{i=1}^{v} \tau_{i}, \\
\text { subject to } \sum_{i=1}^{V} x_{i j}=1, \quad j \in V, j \neq 1, \\
\sum_{j=1}^{V} x_{i j}=1, \quad i \in V, i \neq 1, \\
\sum_{i=1}^{V} x_{i p}-\sum_{j=1}^{V} x_{i p}=0, \quad p \in v, p \neq 1, \\
\sum_{j=2}^{V} x_{1 j}=1, \\
\sum_{i=2} x_{i 1}=1, \\
T_{M N} \leq T_{D},
\end{gathered}
$$

$$
L_{\text {sum }} \leq L_{\text {max }} .
$$

Equations (7) and (8) ensure that each cluster head node is accessed only once per round. Equation (9) guarantees tour continuity. Equation (10) guarantees that the MS starts from the base station. Equation (11) guarantees that the final destination of MS is the base station. Equation (12) is the time limit per round. Equation (13) is the limit of tour length per round, which in turn determines the energy limit.

Since the MS consumes the most energy on traversing through the sensing field, the energy cost is closely related to the tour range. This paper mainly takes energy expenditure on traveling into account. Therefore, the objective function in optimization is replaced by the target to minimize the tour length as follows:

$$
\operatorname{Minimize} \sum_{i=1}^{V} \sum_{j=1}^{V} x_{i j} d_{i j} \text {. }
$$

\section{Tour Planning}

3.1. Coverage Issue with an Adjacency Matrix. The coverage issue is essential for a WSN. On the one hand, visiting fewer nodes will reduce the MS burden for data collection. On the other hand, a WSN needs enough nodes to achieve the expected monitoring quality. Thus, there is a balance to strike between the node number and the communication quality. In this paper, the network coverage with nodes deployment in random order is concerned. For simplicity purpose, the optimal density of the 
deployment is determined by the communication radius, which is the basis for calculating the number of deployment nodes.

This paper uses an adjacency matrix graph to define the connectivity of deployed nodes [22-26]. We consider the network as a graph $G=(V E)$ where $V$ refers to the SNs and $E$ represents the edge between the two SNs which can communicate with each other.

In some conditions, we can convert $G$ into a square matrix $A=\left(a_{i j}\right)_{m \times m}$ with M-order $(m=|V|)$ if $a_{i j}=\left|e_{k}\right|, e_{k}=\left\langle v_{i}, v_{j}\right\rangle \in E$. Therefore, the adjacency matrix $G$ can be represented by matrix A.

Furthermore, we define $A^{k}=\left(a_{i j}^{(k)}\right)_{m \times m}$, $\left(a_{i j}^{(k)}\right)=\sum_{k=1}^{m} a_{i h}^{k-1} a_{h j}$. If $a_{i h} \bullet a_{h j} \neq 0, a_{i h} \neq 0$, and $a_{h j} \neq 0$, there are $k$ routes from the node $v_{i}$ via $v_{h}$ to $v_{j}$. For instance, $a_{i j}^{(2)}$ demonstrates there are two paths that can start from $v_{i}$ via an intermediate node $v_{h}$ to the node $v_{j}$. Therefore, if $\sum_{k=1}^{m-1} a_{i j}^{(k)}=0$, there is no path communication between the node $v_{i}$ and the node $v_{j}$.

Therefore, we use a new matrix $S=\left(S_{i j}\right)_{m \times m}=\sum_{k=1}^{m-1} A^{k}$. If all the elements in matrix $S$ are nonzero elements, then $G$ is a fully connected graph. Otherwise, if there is only an element in matrix $S$, then $G$ is a disconnected graph.

3.2. Cluster Forming. We uniformly deploy the nodes in the region where they are grouped into clusters. In this way, the SNs can have balanced energy consumption since they can share the workload in clusters $s$ [27]. Moreover, it ensures that each cluster head has almost the same energy expenditure in each cluster. There are many useful and advanced algorithms to realize the deployment, for example, neighbor clustering and fuzzy clustering. In order to implement real-world tests, we want a simpler and efficient algorithm as our solution. Therefore, the $K$-means algorithm is adopted. We use $K$-means based dynamic clustering algorithm to partition the nodes into the monitoring area [24].

The $K$-means algorithm aims to minimize the total distance $J_{j}$ between the SNs and the center of the cluster. The distance can be presented by

$$
J_{j}=\sum_{i=1}^{N_{j}}\left\|X_{i}-Z_{j}\right\|^{2}, \quad X_{i} \in S_{j},
$$

where $S_{j}$ is cluster $j ; Z_{j}$ is the center of the cluster $j$; and $N_{j}$ is the sample number in the cluster $j$. Since the selection of the cluster center should make $J_{j}$ extremely small [1], then $\partial J_{j} / \partial Z_{j}=0$. Thus, equation (14) can be rewritten by

$$
\frac{\partial}{\partial_{Z j}} \sum_{i=1}^{N}\left\|X_{i}-Z_{j}\right\|^{2}=0 .
$$

Therefore,

$$
Z_{j}=\frac{1}{N_{j}} \sum_{i=1}^{N_{j}} X_{i}
$$

The algorithm is committed in the following way:

Step 1: initializing the clusters with randomly picked nodes. A cluster head is selected to be as the center. All centers are recorded by $Z_{1}(1), Z_{2}(1), \ldots \ldots \ldots, Z_{k}(1)$.

Step 2: allocating the rest of the SNs in cluster $k$ around the centers according to equation (17) where Euclidean distance is applied.

Step 3: recalculating $Z_{j}(k+1), j=1,2, \ldots, k$ for each cluster.

Step 4: judging if $Z_{j}(k+1)=Z_{j}(k)$, the clustering ends. Otherwise, return to Step 2 and regroup the sample iteratively.

Afterwards, the calculation completes while the results are recorded. Now, the nodes are partitioned to $k$ clusters where $k$ virtual cluster centers are generated at the same time. Via $k$-means algorithm, the overall distance from the $\mathrm{SNs}$ to the cluster centers reaches the least. Since the intercluster communication uses single-hop transmission, the communication costs the least energy consumption through this arrangement. The nodes are then manually placed to the locations of these virtual cluster centers where the cluster head nodes play the roles of the center. After a head node being defined, a broadcast message is sent to the members in the cluster to confirm the location of the cluster head node. Now, the location of these virtual cluster centers will be a rendezvous point for the MS. The MS starts to gather data by the members in the cluster after the MS reaches the location.

3.3. Energy Consumption Prediction. Due to the limited energy capacity of the MS, it is difficult for a single MS to complete all the data gathering without interruption in one cycle. Therefore, the whole tour planning is divided into several segments where the division method is defined by the prediction result based on the ant colony system (ACS) optimization. The algorithm follows the steps below:

Step 1: to determine the accessible order of each cluster where the access nodes are defined by $\{1,2, \ldots, v\}$. These orders are determined by the ant colony algorithm [8-27]. The calculation is based on equations (18)-(20).

Step 2: judgement. If the length of the whole tour is within the maximum range of MS, the MS completes the data gathering at once. Otherwise, the tour is cut into multiple segments and executed in turn. 
Assuming $j$ represents the next hop point from the current $i$ point, the possibilities of visiting point $j$ are $s$ that can be presented by the following equations [27]:

$j=\left\{\begin{array}{l}\arg \max \left\{\left[\tau_{i j}(t)\right]\left[\eta_{i j}(t)\right]^{\beta}\right\}, \quad q<q_{0}, \\ S,\end{array}\right.$

$S= \begin{cases}\frac{\left[\tau_{i j}(t)\right]\left[\eta_{i j}(t)\right]^{\beta}}{\sum_{k \in \text { allowed }_{k}}\left[\tau_{i j}(t)\right]\left[\eta_{i j}(t)\right]^{\beta}}, & j \in \text { allowed } k \\ 0, & \text { else }\end{cases}$

where $\tau_{i j}(t)$ represents pheromone variables; $\eta_{i j}(t)$ is the heuristic desirability; $\beta>0$ represents the relative strength of heuristic factors; $q_{0} \in(0,1)$ is the initial parameters; and $q \in(0,1)$ is a random number. Local update strategy is to make the selected edges have a better influence on the later ants, as shown in the following formula:

$$
\tau_{i j}(t+1)=(1-\zeta) \tau_{i j}(t)+\zeta \cdot \tau_{0},
$$

where $\zeta$ is the pheromone decay parameter within $(0$, $1)$ and $\tau_{0}$ is a constant. The global update strategy is to find the shortest path for traversing the rest of the points and return to the base station within the MS energy capability that belongs to the edges on the optimal path, as shown in the following equation:

$$
\begin{aligned}
\tau_{i j}(t+1) & =(1-\rho) \tau_{i j}(t)+\rho \cdot \Delta \tau_{i j}(t), \\
\Delta \tau_{i j} & = \begin{cases}\frac{1}{L_{k}}, & (i, j) \text { on the optimal path, } \\
0, & \text { else, }\end{cases}
\end{aligned}
$$

where $\Delta \tau_{i j}$ is the added pheromone on the trail from point $i$ to $j ; \rho$ represents the evaporation coefficient, $\rho \in(0,1)$; and $L_{k}$ is the optimal path length.

Step 3: the total energy carried by the MS is $E$. At the beginning of the cycle, the MS arrives at the first sensor node for data collection. Afterwards, the MS measures its own remaining energy for the next stage. If the remaining energy is enough to be consumed in the next stage, the MS travels to the next node for data gathering. Otherwise, it returns directly to the base station for recharging. The energy consumption in the next stage is expected to be sufficient for visiting the remaining nodes, data collecting, and returning to the base station.

(1) Energy consumption for visiting:

It refers to the energy consumed over the traveling from node $i$ to node $j$ :

$$
e_{i j}=e_{m} \bullet d_{i j}
$$

(2) Energy consumption for collecting data from node $j$ :

$$
e_{\text {rej }}=r e_{r x} \bullet\left(t_{j}-t_{0}\right) \text {. }
$$

$t_{j}$ is the time to arrive at node $j$, and $t_{0}$ is the starting time of the cycle.

(3) Energy consumption for returning to the base station:

$$
e_{j s}=e_{m} \bullet d_{j s}
$$

The total energy consumption for the next stage is predicted at node $i$ expressed by the following equation:

$$
N_{i}=e_{i j}+e_{\text {rej }}+e_{j s}
$$

Assuming the remaining energy when the MS reaches the node $i$ is equal to $q$, the remaining energy of the MS after the completion of the data gathering is $R_{i}$. So,

$$
R_{i}=Q-e_{\text {rei }}-e_{\text {chi }}
$$

If $R_{i}>N_{i}$ when MS travels to node $j$ for data collection, MS goes on its journey to the next node. Otherwise, the MS returns to the base station for recharging.

Step 4: tour replanning.

After the MS completes the recharge, the tour planning to the remaining nodes in the next subcycle will be recalculated. The new trajectory and visiting order are calculated. Afterwards, the data gathering continues according to the updating results from Step 2.

Step 5: repeat the above process until all the cluster fields are traversed. The remaining nodes not polled are abandoned. In order to simplify the algorithm, the maximum tour length is used to represent the energy limit of the MS.

\section{Results and Analysis}

4.1. Simulation Environment. To verify the effectiveness of the algorithm, the simulation is carried out on a MATLAB platform. The key simulation parameters are summarized in Table 1.

4.2. Outcomes and Analysis. First, the problem of node coverage is simulated. Assuming the node number is 90 and the communication radius is $15 \mathrm{~m}$, the coverage area is shown in Figure 2. After a large number of experiments being committed, the relationship between node number, communication radius, and coverage are obtained. The results are shown in Figure 3. The relationship between node density and coverage is shown in Figure 4. By synthetically analyzing these outcomes, we define the 
TABLE 1: Parameters of the simulation.

Parameter name Parameter values

Number of sensor nodes

Number of virtual cluster head nodes

Number of MS

Heuristic factor $(\alpha)$

Expectation heuristic factor $(\beta)$

Information intensity $(Q)$

Pheromone volatile factor $(\eta)$

70

Number of ant colonies $(m)$

Required coverage (\%)

Maximum endurance mileage $(m)$

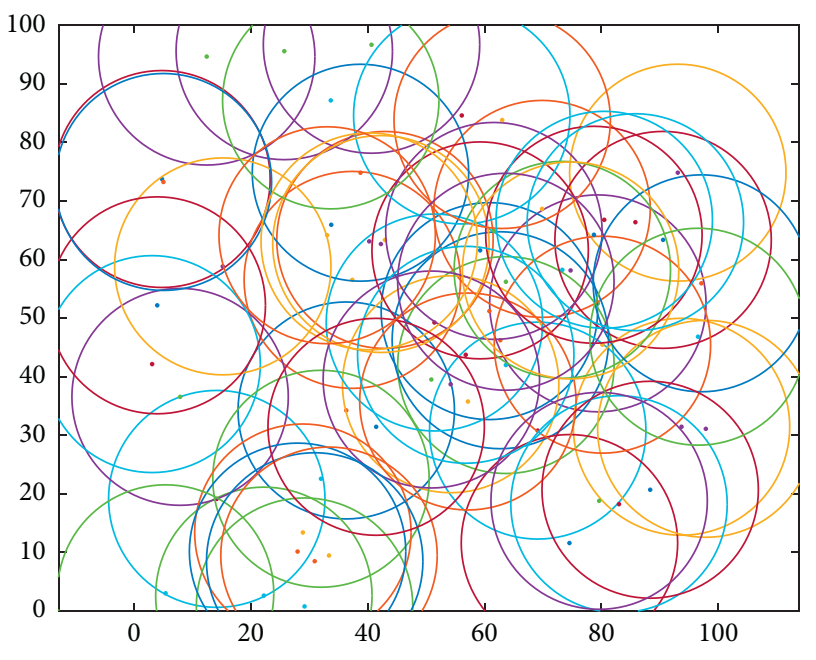

FIgURE 2: Coverage area in the field.

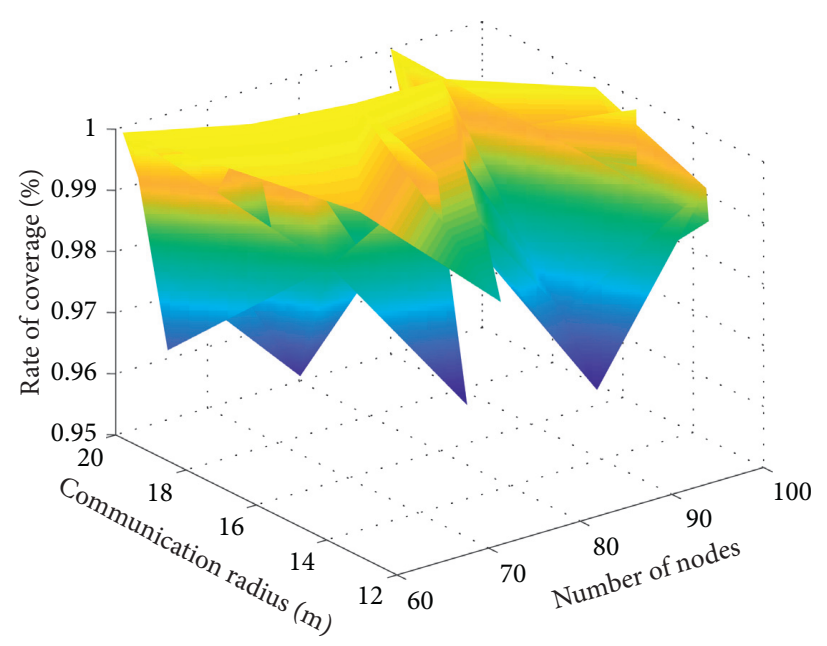

FIgURE 3: Relationship between the number of nodes, communication radius, and coverage.

communication radius and the node density as 15 and 0.007, respectively.

According to the coverage plan, 70 nodes are randomly placed in the sensor field as shown in Figure 5. After applying the dynamic clustering algorithm, 10 virtual cluster head nodes are generated as shown in Figure 6.
Afterwards, the ant colony optimization is used to plan the shortest path over the ten cluster head nodes, and the node path is shown in Figure 7. However, the shortest length on the tour is 348.63 according to the measurement from Figure 8. It exceeds the energy limit of the MS. The access order can be obtained as shown in Figure 9. Nevertheless, the 


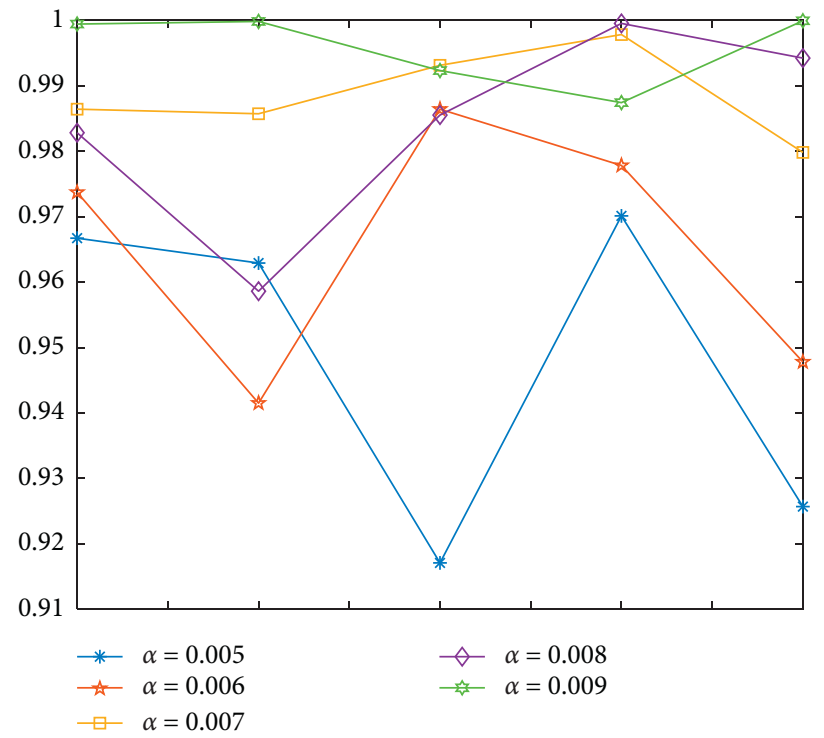

Figure 4: Relationship between node density and coverage ( $r=15)$.

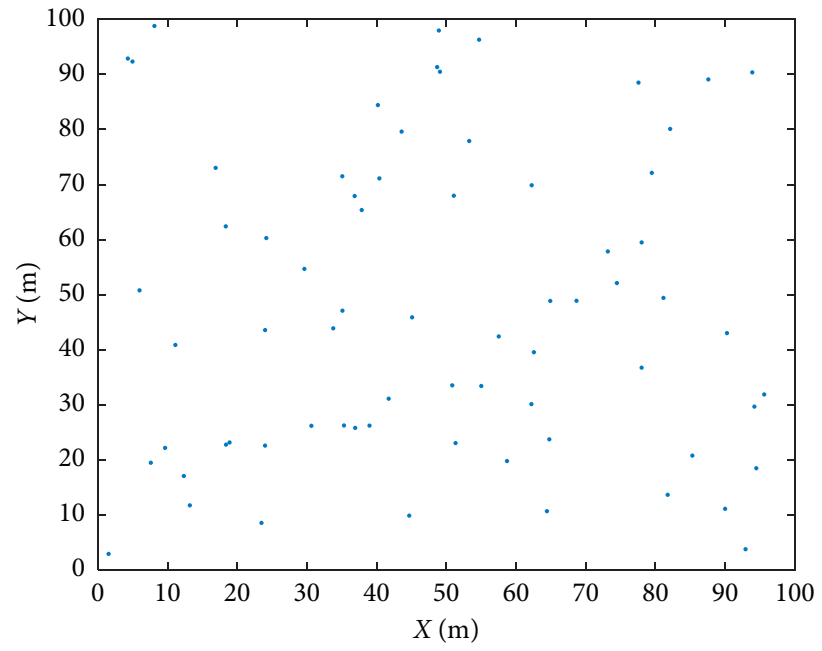

Figure 5: Node distribution.

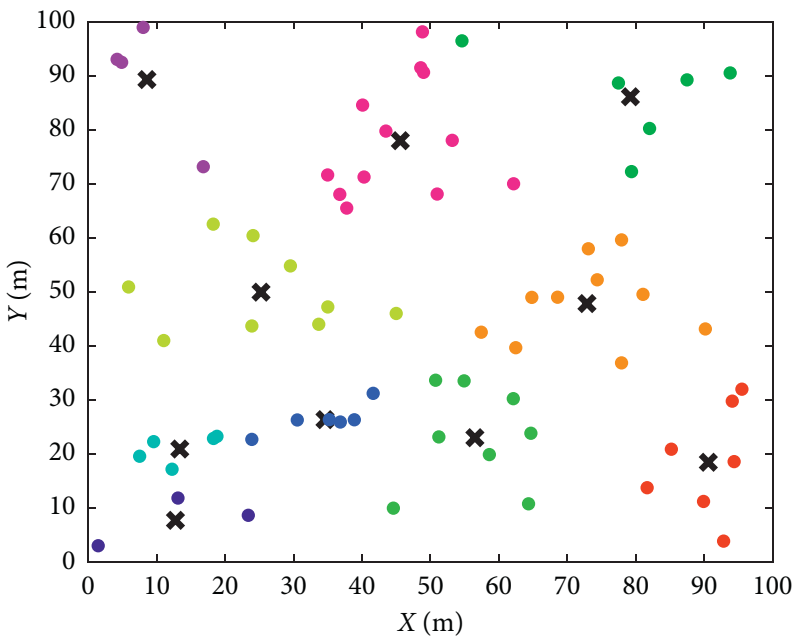

FIgURE 6: Node clustering and the cluster heads. 


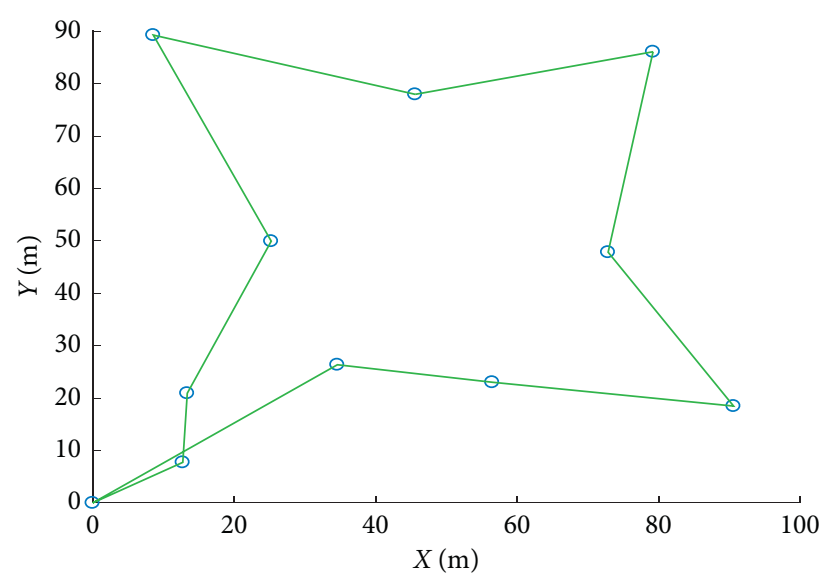

FIgURE 7: The planned trajectory of a MS at the beginning.

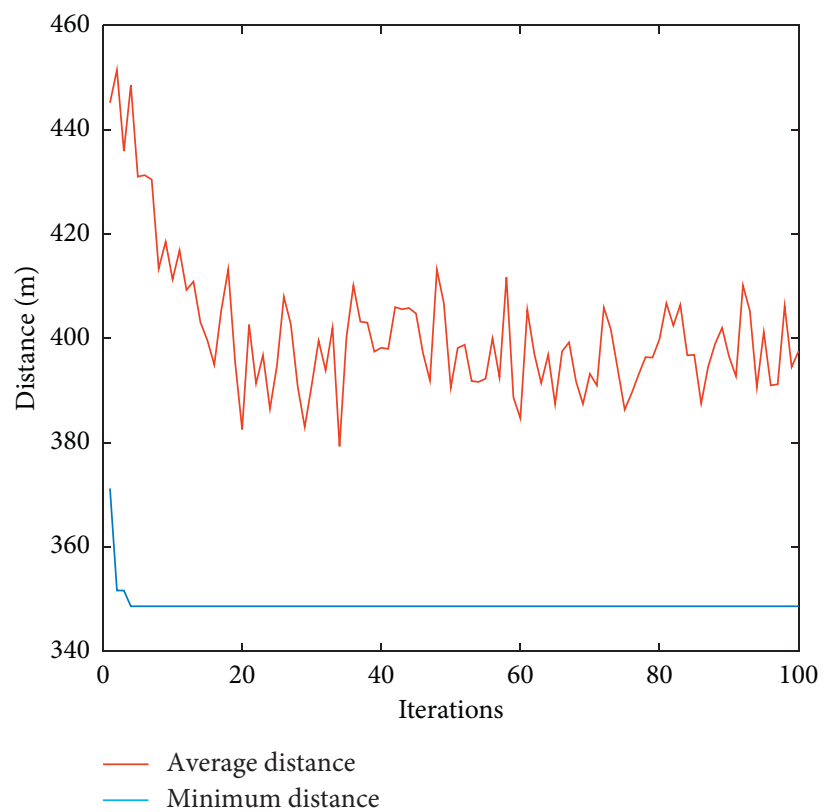

Figure 8: Predicted path distance based on prediction.

node starts the data gathering for the first subcycle from the base station (node 0 ).

The MS starts the tour. However, when it reaches node 3, it is found that the remaining energy is not enough to support it to complete the next stage of data collection tasks; that is, it reaches node 4 for data collection tasks and returns to the base station. Thus, it returns to the base station for energy replenishment to continue the second subcycle. After the MS is fully charged, the tour planning for traversing the remaining nodes is recalculated as shown in Figure 10. The shortest path is shown in Figure 11. At this time, traversing the remaining nodes costs more energy than the MS capacity. There will be a third subcycle for the MS. The updated access order in the second subcycle can be found in Figure 12.

Again, the MS arrives at the head node of the first virtual cluster in the second subcycle. After node 3 being poll, the MS needs more energy to reach node 4 . Therefore, it returns to the base station for preparing the third subcycle.

After being fully charged, the remaining tour is reorganized. The third subcycle is then defined as shown in Figure 13. The searching map for the optimal trajectory is shown in Figure 14. The shortest length over the field cannot be completed by the MS in one cycle. Therefore, the fourth subcycle is needed. The calculation for the third subcycle with the optimal is executed and the result is shown in Figure 15. 


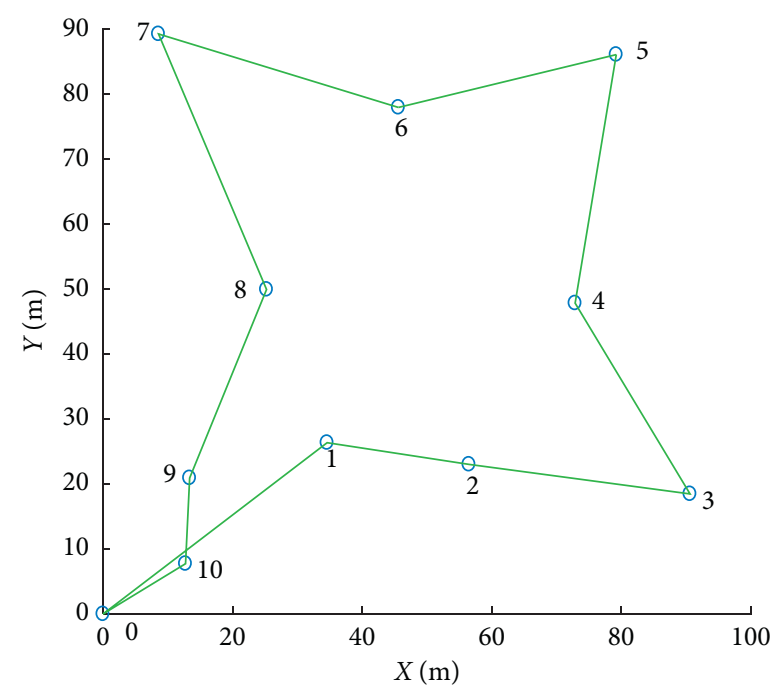

Figure 9: Planned access order for the first round prediction.

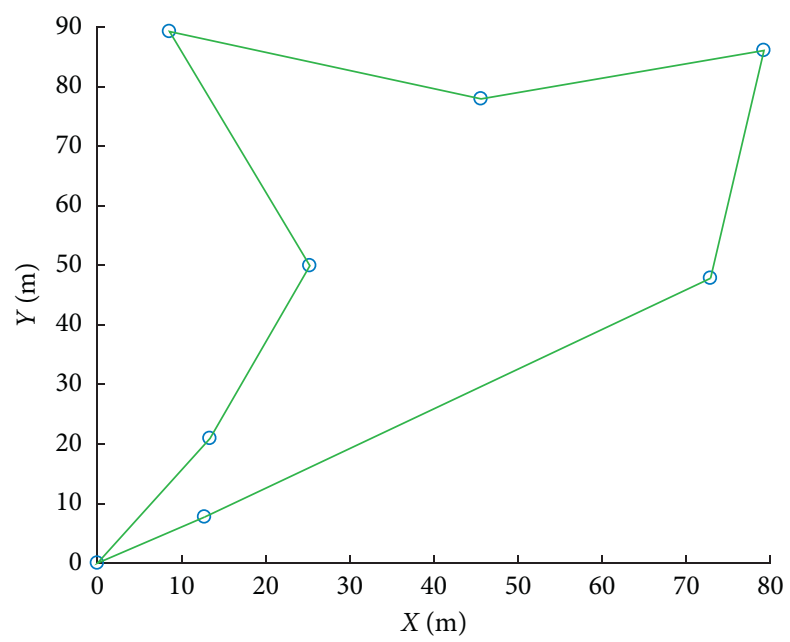

Figure 10: Planned trajectory at the beginning of the second subcycle.

The plan for the traversing, the predicted path length, and the node access order of the fourth subcycle are shown in Figures 16-18

In the fourth subcycle, it is slightly different from the previous subcycles. After polling node 2, the MS cannot reach node 3 . It returns to the base station to be recharged. However, the energy cannot support it traveling to node 3. Therefore, node 3 is abandoned. The tour planning, trajectory, and the visiting order for the fourth subcycle are demonstrated in Figures 19 and 20.

The simulation results have shown the initial energy of the MS cannot support completing data collection. Therefore, the initial energy is then increased to 300 . By repeating the process above, the tour planning in the final two periods can be obtained as shown in Figure 21. The path lengths of each subcycle are shown in Figure 22. With the updated initial energy, the MS can complete the data gathering by traversing all cluster head nodes over the sensing field. Therefore, the optimal energy capacity of the MS is determined by $300 \mathrm{~m}$.

The simulation is carried out with the optimal tour planning with the shortest length where energy constraints and efficient data gathering are considered as a priority. Through the simulation, we also find the process of data gathering is closely related to the initial energy of the MS. 


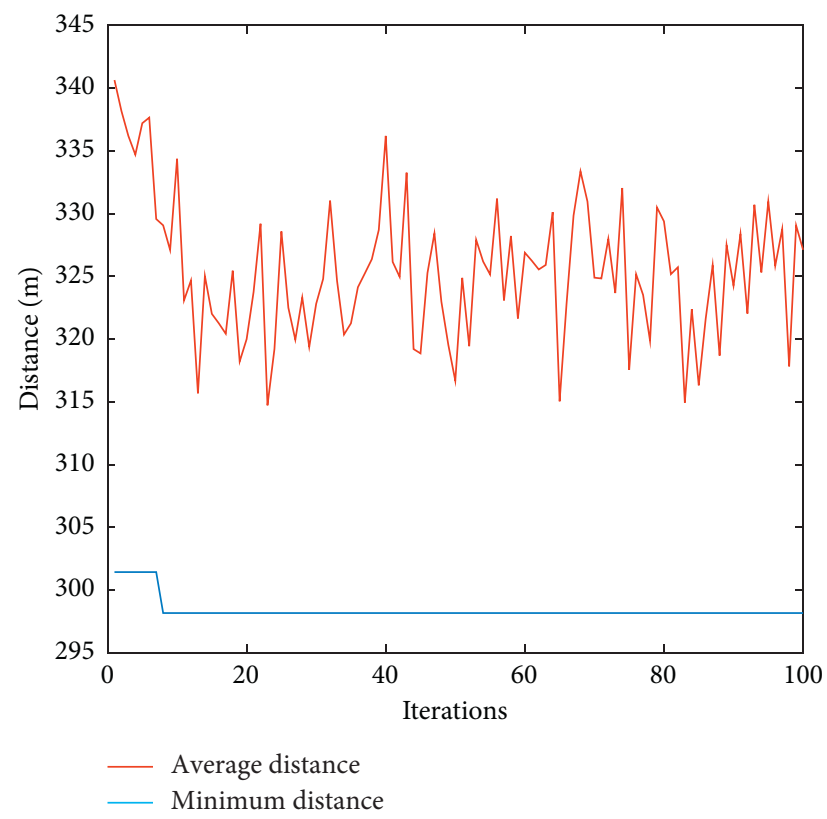

Figure 11: Predicted path length at the beginning of the second subcycle.

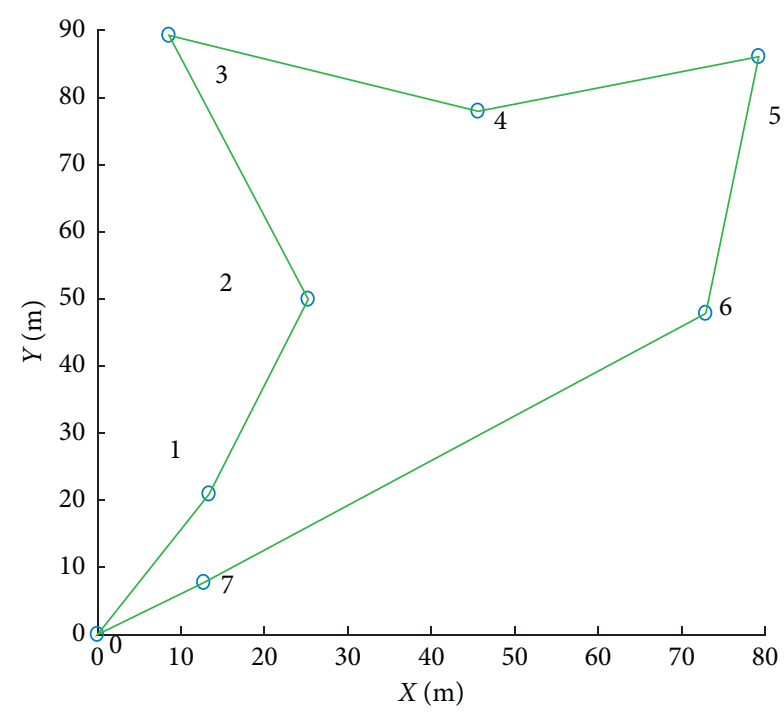

Figure 12: Planned access order in the second subcycle.

Therefore, a comparative evaluation for the relationship between the tour planning and battery capacity of the MS is carried out. The key indicators are summarized in Table 2.

It is noted that the larger initial energy can lead to a smaller number of subcycles. Moreover, the shorter length means the shorter latency over data gathering.

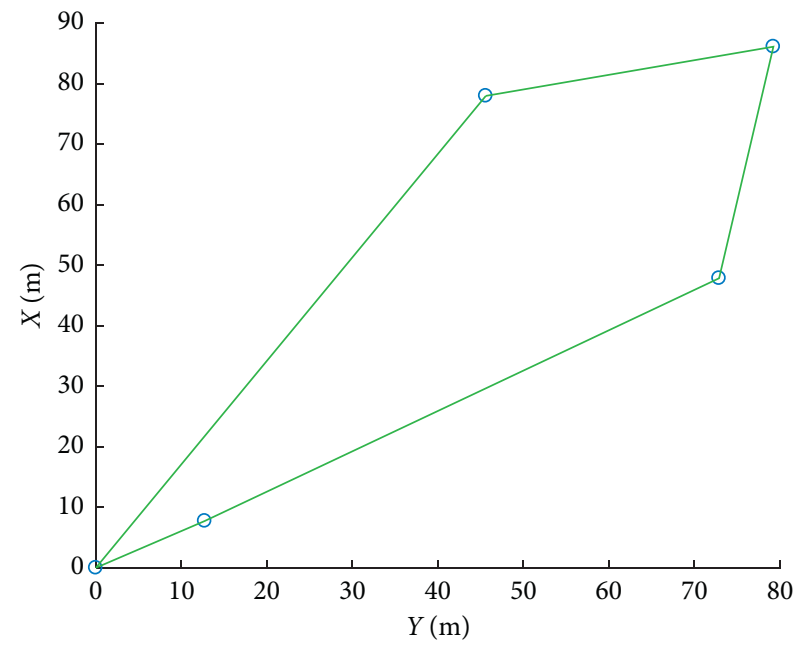

Figure 13: Planned trajectory at the beginning of the third subcycle.

In practice, the energy capacity of a MS is limited. Therefore, consideration is needed to strike the balance between the delay and the initial energy.

4.3. Trail Tests in a Small WSN. Trail tests are carried out in the laboratory to verify the reliability of data transition based on a small scale WSN while only the first subcycle is tested. Three SNs are placed as the terminal nodes to collect data. 


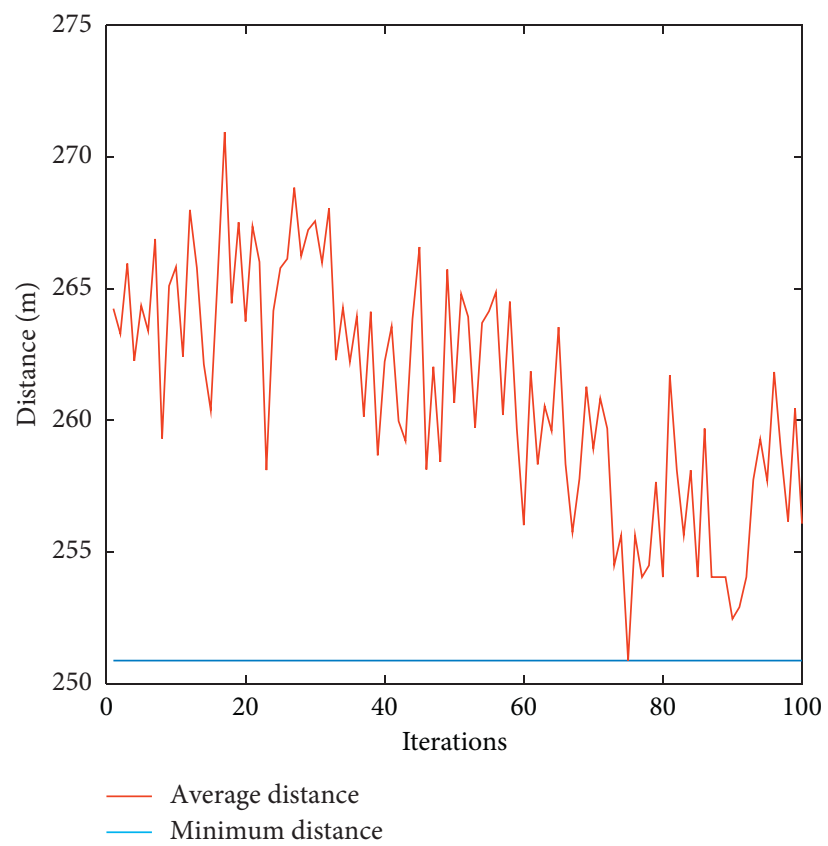

FIgURE 14: Predicted path length at the beginning of the third subcycle.

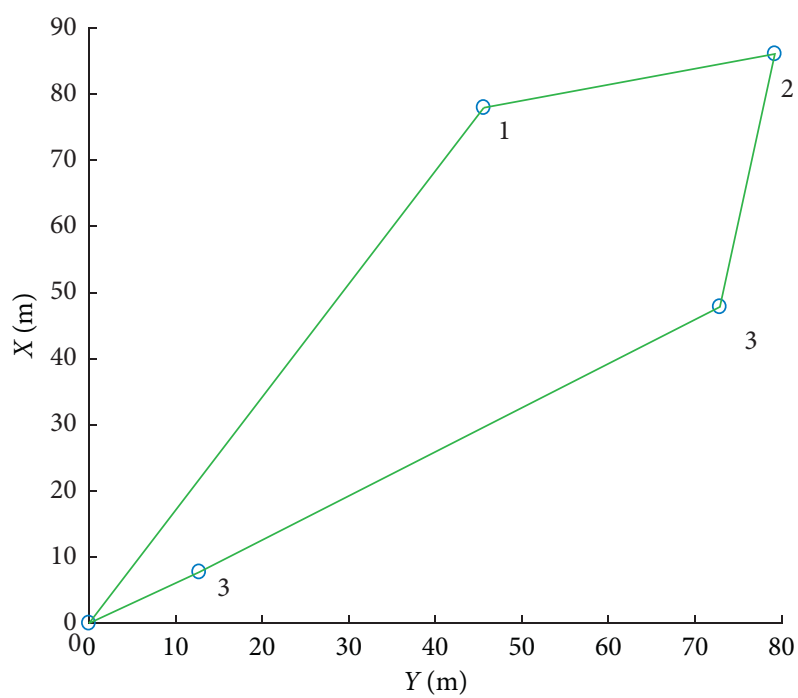

Figure 15: Planned access order at the beginning of the third subcycle.

The MS is responsible for polling the SNs and completes data gathering. The device in the experiment includes a UAV carrying the MS and three DHT11 temperature-humidity sensors as the SNs. The images of these devices are shown in Figure 23. Test results are summarized in Table 3.
Furthermore, three rounds of experiments are committed. The results are summarized in Table 4 . The results show that the experimental system can effectively collect temperature and humidity data, and the data transmission is reliable. 


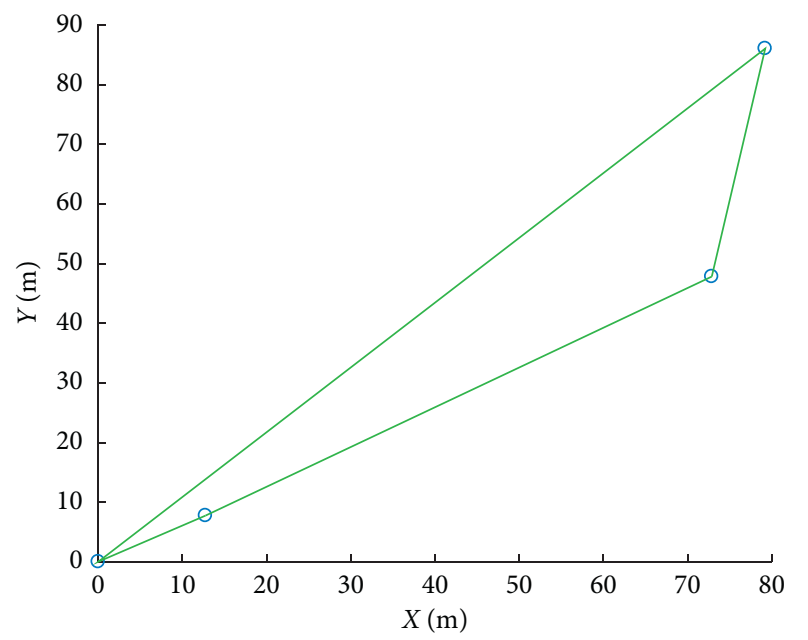

Figure 16: Planned trajectory at the beginning of the fourth subcycle.

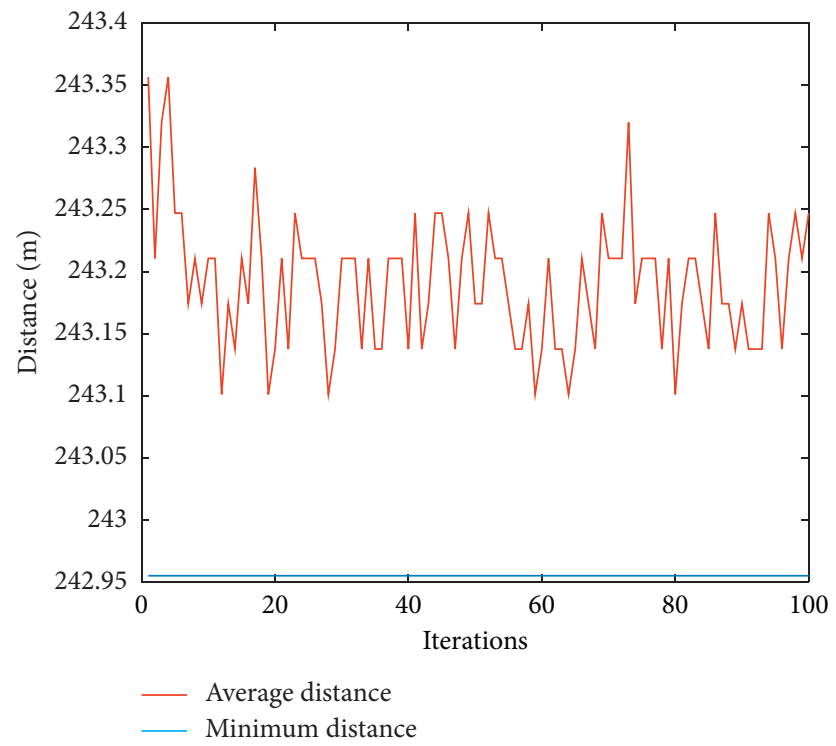

FIgURE 17: Predicted path length at the beginning of the fourth subcycle.

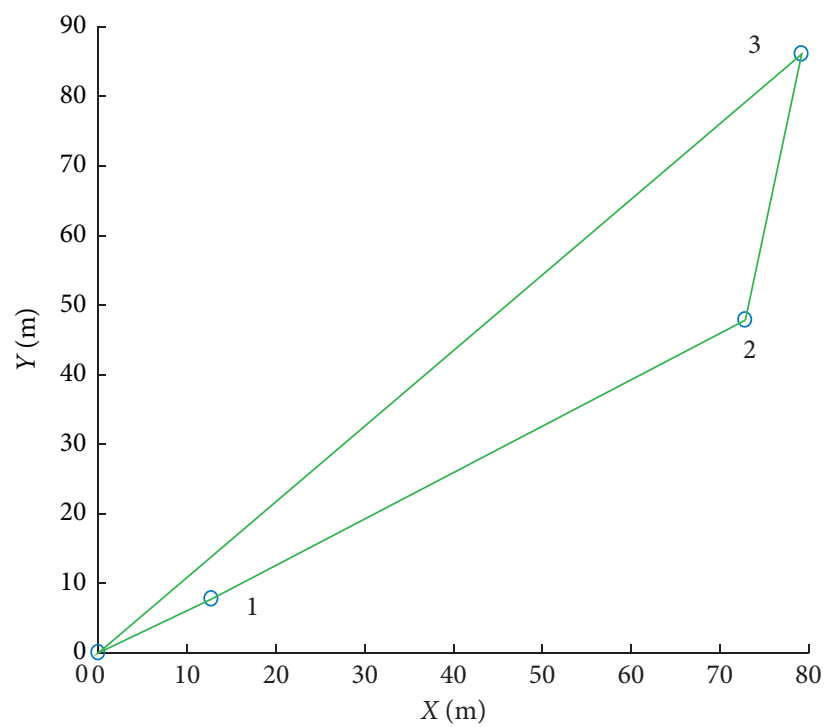

FIGURE 18: Planned access order at the beginning of the fourth subcycle. 


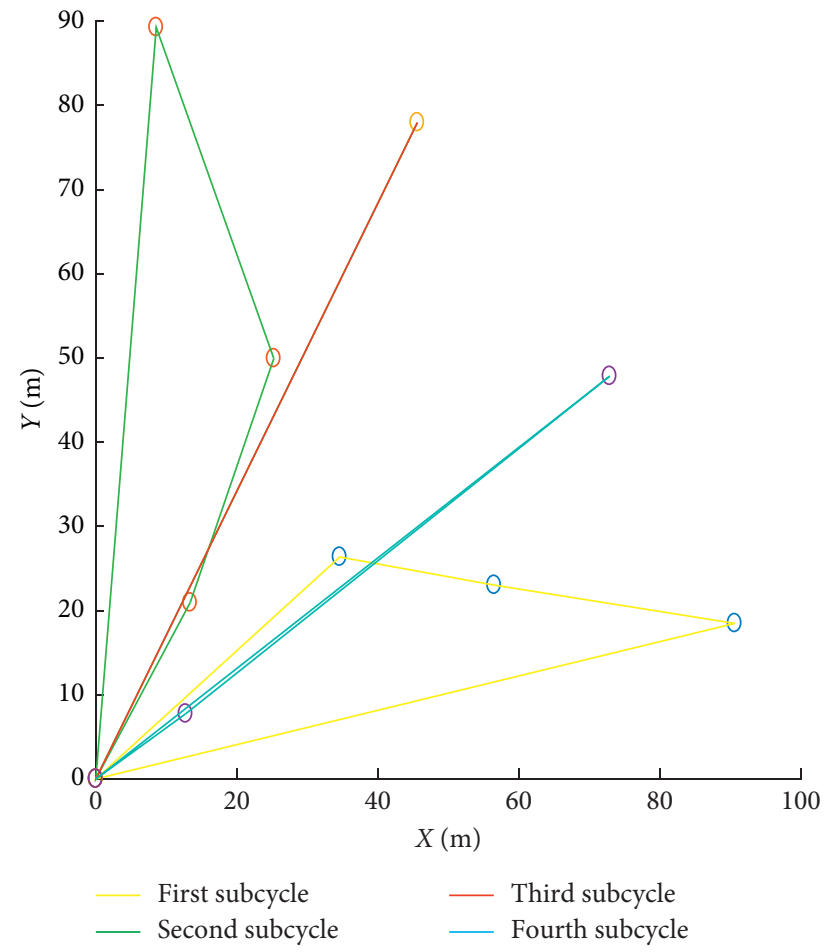

Figure 19: The whole tour planning over the four subcycles.
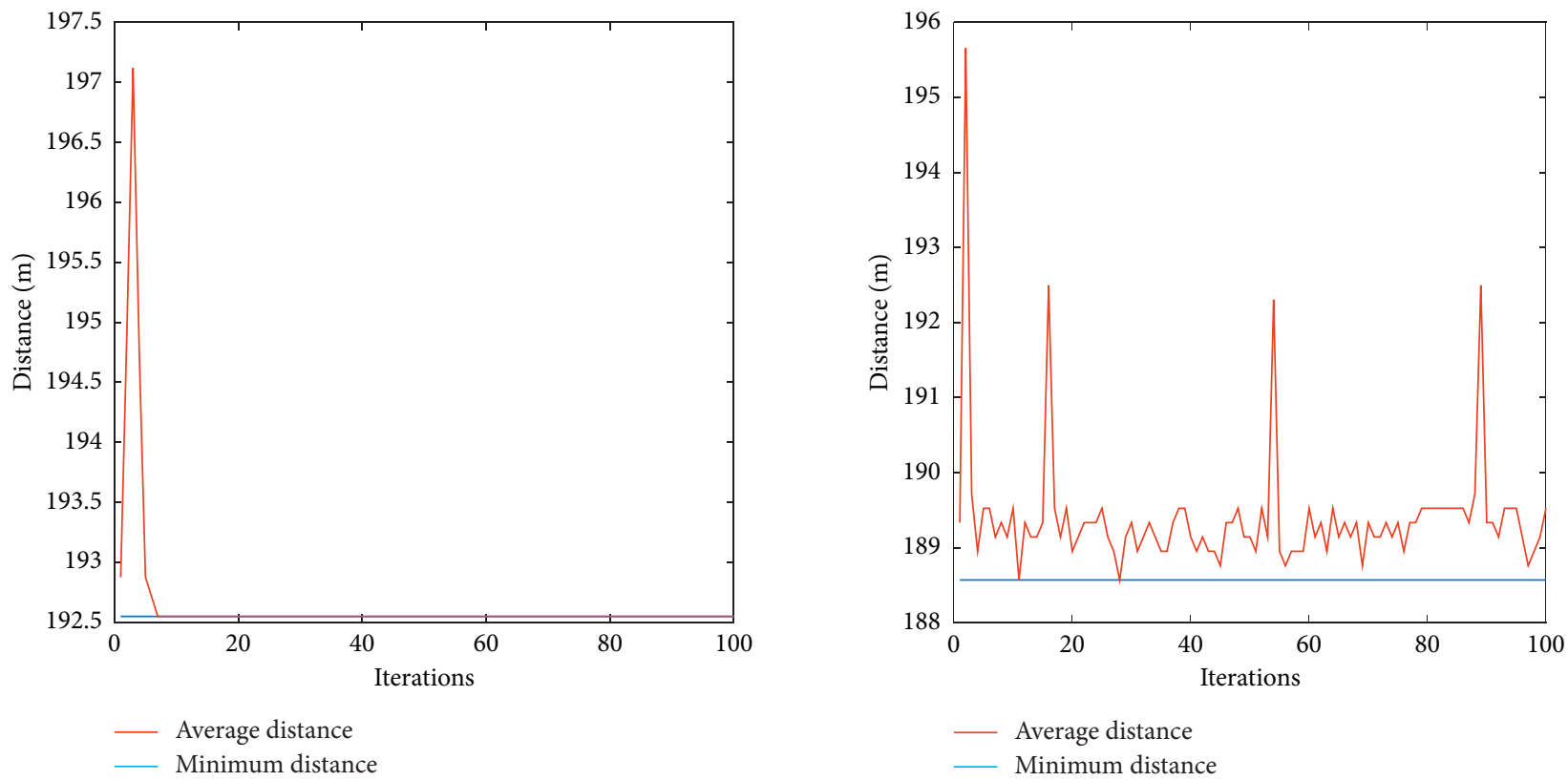

(a)

(b)

FIGURE 20: Continued. 


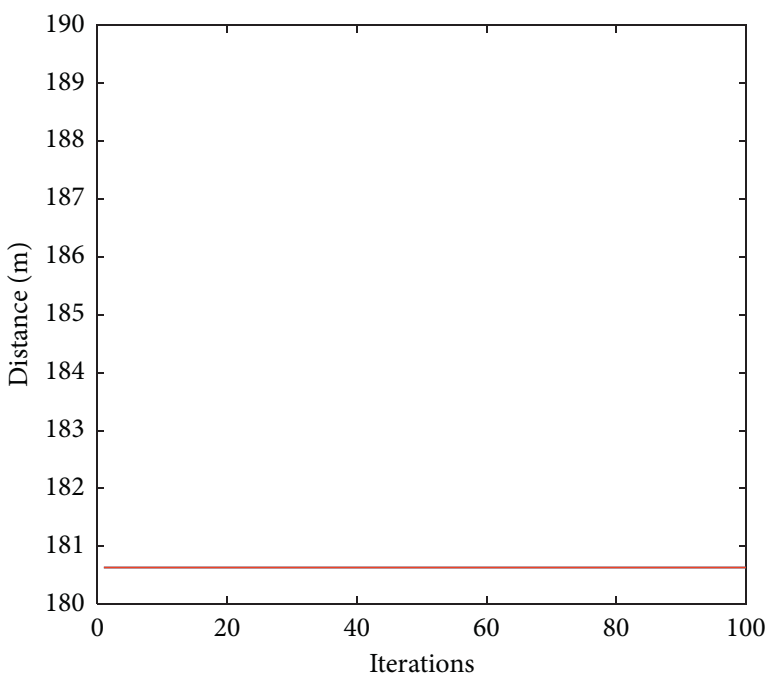

Average distance Minimum distance

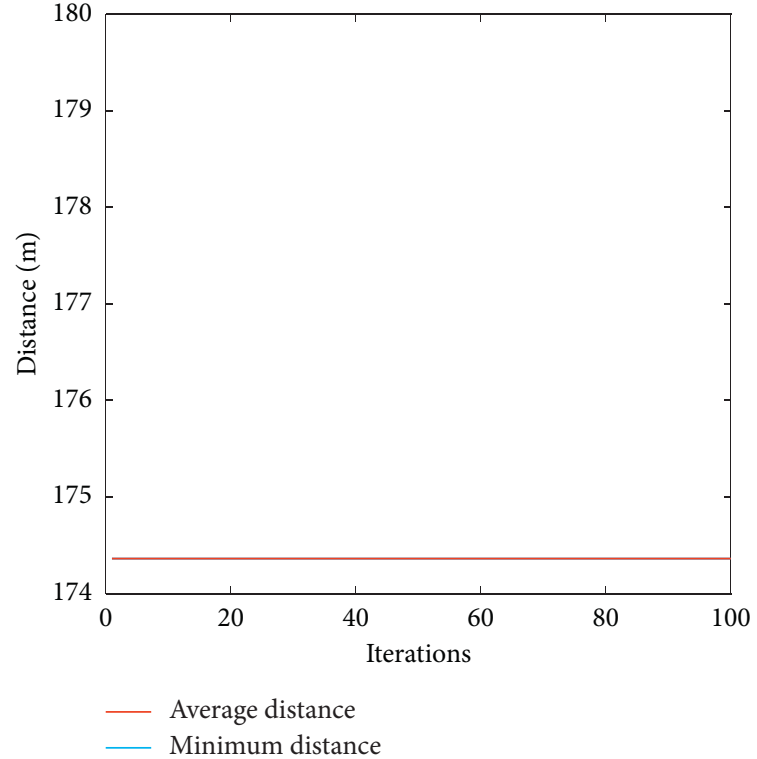

(d)

Figure 20: Tour length for each subcycle.

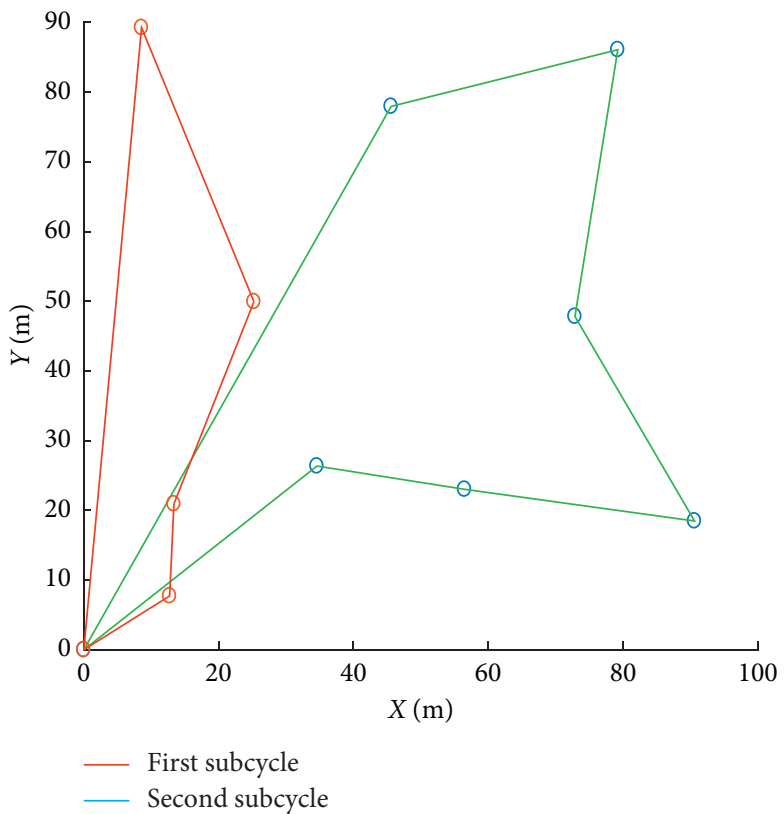

Figure 21: The first two subtours with a limitation of a maximum continuation length of 300 meters. 


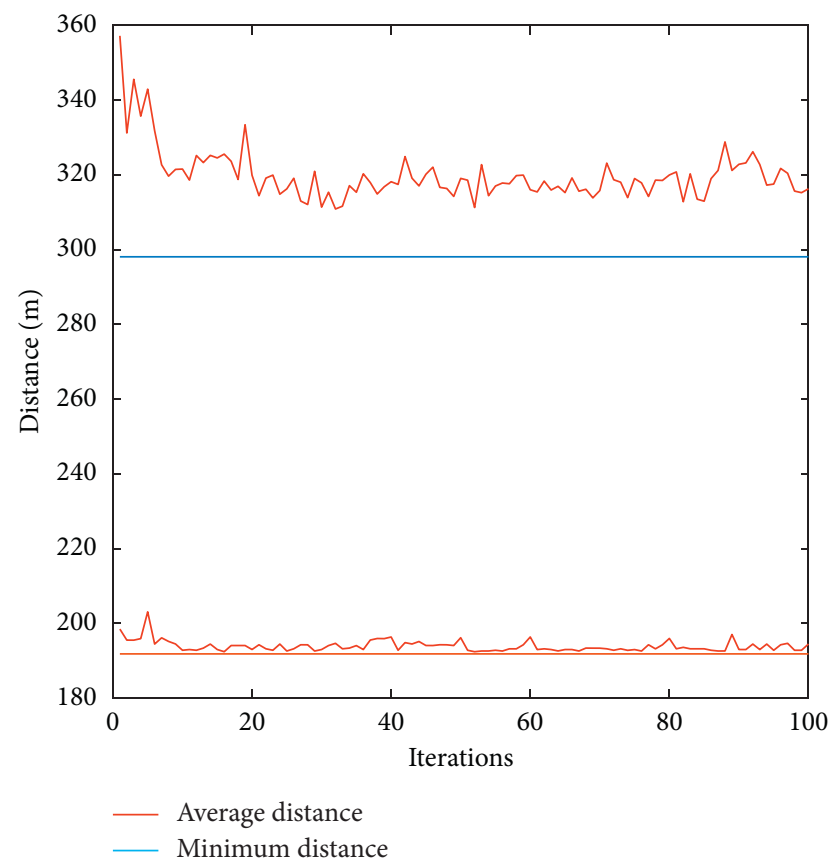

Figure 22: The path length of the first two subtours with a limitation of a maximum continuation length of 300 meters.

TABLE 2: Tour planning with different battery capacities.

\begin{tabular}{lcccc}
\hline Round & Maximum range $(\mathrm{m})$ & Number of cycles & Data gathering rate $(\%)$ & Total length of the route $(\mathrm{m})$ \\
\hline 1 & 200 & 4 & 90 & 736 \\
2 & 300 & 2 & 100 & 490 \\
3 & 400 & 1 & 100 & 350 \\
\hline
\end{tabular}

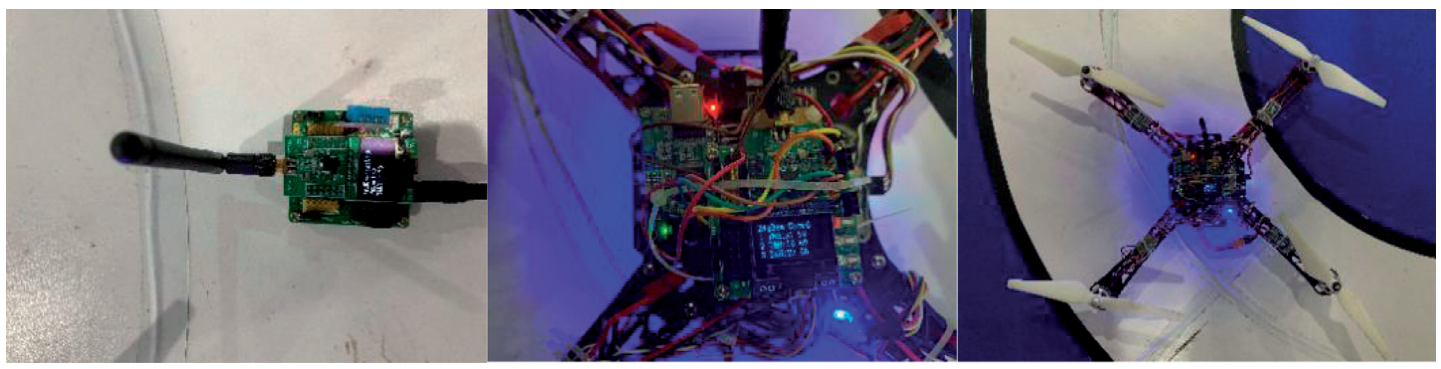

FIGURE 23: Device for sensing and data gathering.

TABLE 3: Test results of communication quality.

\begin{tabular}{lccc}
\hline Distance $(\mathrm{m})$ & Number of packets sent & Number of packets lost & Packet loss rate \\
\hline 10 & 1000 & 0 & 0 \\
15 & 1000 & 0 & 0 \\
20 & 1000 & 1 & 0.001 \\
25 & 1000 & 23 & 0.022 \\
30 & 1000 & 101 & 0.121 \\
35 & 1000 & 382 & 0.394 \\
40 & 1000 & 1000 & 1 \\
\hline
\end{tabular}

TABLE 4: The experimental values of the system.

\begin{tabular}{lccc}
\hline Number of experimental rounds & Node 1 & Node 2 & Node 3 \\
\hline 1 & $21 / 59$ & $19 / 69$ & $22 / 56$ \\
2 & $24 / 50$ & $24 / 55$ & $24 / 51$ \\
3 & $22 / 53$ & $21 / 59$ & $24 / 51$ \\
\hline
\end{tabular}




\section{Conclusion}

This paper presents a prediction-based tour planning for data gathering in WSNs. The scheme primarily aims to mitigate the challenge when a single MS cannot complete data gathering or traverse all cluster head nodes at once. First, the issue of the network coverage is discussed in favor of a decent arrangement with the optimal number of sensor nodes across the field. Afterwards, a dynamic clustering algorithm is proposed to generate sensor clusters where the cluster head nodes are defined as the MS sojourns. The methodology of the predictive trajectory is developed for finding the optimal tour before a MS traverses the sensing field. In the study, the single tour of data gathering is divided into several subcycles to prevent energy exhaustion. Both the MATLAB simulation and physical tests are committed to demonstrating the whole procedure for data gathering under the proposed scheme. The results have proved the effectiveness and reliability of the methodology. The study outcomes provide a useful solution for tour planning in energy-constrained WSNs.

\section{Data Availability}

The data used to support the findings of this study are included within the article. If other data or programs used to support the findings of this study are needed, they can be obtained from the corresponding author upon request.

\section{Conflicts of Interest}

The authors declare that they have no conflicts of interest.

\section{Acknowledgments}

This work was supported partly by the National Natural Science Foundation of China (61861007).

\section{References}

[1] X. Li, J. Peng, M. S. Obaidat, F. Wu, M. K. Khan, and C. Chen, "A secure three-factor user authentication protocol with forward secrecy for wireless medical sensor network systems," IEEE Systems Journal, vol. 14, no. 1, pp. 39-50, 2020.

[2] H. Malik and W. Zatar, "Agent based routing approach to support structural health monitoring-informed, intelligent transportation system," Journal of Ambient Intelligence and Humanized Computing, vol. 11, no. 3, pp. 1031-1043, 2020.

[3] D. Achilles, S. Maria, P. Papadopoulou, and K. Goudos, "Internet of things (iot) and agricultural unmanned aerial vehicles (uavs) in smart farming: a comprehensive review," Internet of Things, vol. 92, Article ID 100187, 2020.

[4] A. Demertzis, K. Oikonomou, and I. Stavrakakis, "Evaluation of a proposed minimum path impotence routing policy in wireless sensor networks," Ad Hoc Networks, vol. 94, Article ID 101928, 2019.

[5] N. Sharmin, A. Karmaker, W. L. Lambert, M. S. Alam, and M. S. A. Shawkat, "Minimizing the energy hole problem in wireless sensor networks: a wedge merging approach," Sensors, vol. 20, no. 1, p. 277, 2020.

[6] R. Deng, S. He, P. Cheng, and Y. Sun, "Towards balanced energy charging and transmission collision in wireless rechargeable sensor networks," Journal of Communications and Networks, vol. 19, no. 4, pp. 341-350, 2017.

[7] X. Zhu, X. Tao, T. Gu, and J. Lu, "Target-aware, transmission power-adaptive, and collision-free data dissemination in wireless sensor networks," IEEE Transactions on Wireless Communications, vol. 14, no. 12, pp. 6911-6925, 2015.

[8] K.-C. Chu, D.-J. Horng, and K.-C. Chang, "Numerical optimization of the energy consumption for wireless sensor networks based on an improved ant colony algorithm," IEEE Access, vol. 7, pp. 105562-105571, 2019.

[9] R. Velmani and B. Kaarthick, "An efficient cluster-tree based data collection scheme for large mobile wireless sensor networks," IEEE Sensors Journal, vol. 15, no. 4, pp. 2377-2390, 2015.

[10] Y. Yuan, W. Liu, T. Wang, Q. Deng, A. Liu, and H. Song, "Compressive sensing-based clustering joint annular routing data gathering scheme for wireless sensor networks," IEEE Access, vol. 7, pp. 114639-114658, 2019.

[11] S. Guo, C. Wang, and Y. Yang, "Joint mobile data gathering and energy provisioning in wireless rechargeable sensor networks," IEEE Transactions on Mobile Computing, vol. 13, no. 12, pp. 2836-2852, 2014.

[12] D. Takaishi, H. Nishiyama, N. Kato, and R. Miura, "Toward energy efficient big data gathering in densely distributed sensor networks," IEEE Transactions on Emerging Topics in Computing, vol. 2, no. 3, pp. 388-397, 2014.

[13] L. Nguyen and H. T. Nguyen, "Mobility based network lifetime in wireless sensor networks: a review," Computer Networks, vol. 174, Article ID 107236, 2020.

[14] V. K. Chawra and G. P. Gupta, "Load balanced node clustering scheme using improved memetic algorithm based meta-heuristic technique for wireless sensor network," Procedia Computer Science, vol. 167, pp. 468-476, 2020.

[15] X. Fu and Y. Yang, "Modeling and analysis of cascading nodelink failures in multi-sink wireless sensor networks," Reliability Engineering \& System Safety, vol. 197, Article ID 106815, 2020.

[16] P. Gu, C. Li, Y. Wang, S. Li, and W. Xi, "WSN energy saving strategy with a mobile data collector," Radio Communications Technology, vol. 45, no. 2, pp. 150-155, 2019, (in Chinese).

[17] M. Ma, Y. Yang, and M. Zhao, "Tour planning for mobile data-gathering mechanisms in wireless sensor networks," IEEE Transactions onVehicular Technology, vol. 62, no. 4, pp. 1472-1483, 2013.

[18] F. Liu, H. Lu, T. Wang, and Y. Liu, "An energy-balanced joint routing and charging framework in wireless rechargeable sensor networks for mobile multimedia," IEEE Access, vol. 7, pp. 177637-177650, 2019.

[19] R. Anwit, A. Tomar, and P. K. Jana, "Scheme for tour planning of mobile sink in wireless sensor networks," IET Communications, vol. 14, no. 3, pp. 430-439, 2020.

[20] Z. Meiyan and C. Wenyu, "Dubins curves based mobile data collecting algorithm for clustered wireless sensor networks," Journal Sensor Technology, vol. 32, no. 4, pp. 603-609, 2019.

[21] G. Yogarajan and T. Revathi, "Genetic algorithm-based mobile sink data collecting algorithm for wireless sensor networks," Wireless Network, vol. 24, no. 8, pp. 1095-1099, 2018.

[22] S. Ganesh and R. Amutha, "Efficient and secure routing protocol for wireless sensor networks through SNR based dynamic clustering mechanisms," Journal of Communications and Networks, vol. 15, no. 4, pp. 422-429, 2013.

[23] F. Chao, Z. He, A. Pang, H. Zhou, and J. Ge, "Path optimization of mobile sink node in wireless sensor network water 
monitoring system," Computational Methods for Modeling, Simulating, and Optimizing Complex Systems, vol. 2019, Article ID 5781620, 10 pages, 2019.

[24] A. Pang, F. Chao, H. Zhou, and J. Zhang, "The method of data collection based on multiple mobile nodes for wireless sensor network," IEEE Access, vol. 8, pp. 14704-14713, 2020.

[25] C. Wang, S. Guo, and Y. Yang, "An optimization framework for mobile data collection in energy-harvesting wireless sensor networks," IEEE Transactions on Mobile Computing, vol. 15, no. 12, pp. 2969-2986, 2016.

[26] X. Li, X. Tao, and G. Mao, "Unbalanced expander based compressive data gathering in clustered wireless sensor networks," IEEE Access, vol. 5, pp. 7553-7566, 2017.

[27] P. Zhou, C. Wang, and Y. Yang, "Static and mobile target -coverage in wireless rechargeable sensor networks," IEEE Transactions on Mobile Computing, vol. 18, no. 10, pp. 2430-2445, 2019. 